\title{
A survey of wind engineering studies in India
}

\author{
G N V RAO \\ Department of Aerospace Engineering, Indian Institute of Science, \\ Bangalore 560) (012, India
}

\begin{abstract}
In this paper, the work that has been done in several laboratories and academic institutions in India in the area of wind engineering in the past 20-30) years has been reviewed. Studies on extreme and mean hourly winds, philosophies adopted in model studies in wind tunnels and some of the important results that have been obtained are described. Suggestions for future studies are indicated.
\end{abstract}

Keywords. Extreme wind; industrial structures; aerodynamic loads.

\section{Introduction}

Wind engineering studies, which used to be called industrial aerodynamics at one time, may be said to mean the study of the effects of winds on non-flying structures and vehicles. It includes not only the study of winds in the atmosphere, but also that of wind loads on such earth-fixed structures as chimneys and cooling towers, land transportation vehicles like automobiles, ships and trains, and study of disasters due to wind; it is generally not considered at present to include windmills. pollution, ventilation problems etc., although one may expect in course of time that they may also become part of what is now called wind engineering. It may be said that such studies began in India in 1958, when the Railways requested the Department of Aerospace Engineering. Indian Institute of Science (abbreviated hereafter as $11 \mathrm{sc}$ ), Bangalore, to measure the wind loads on a platform shelter (figure 1) of the type widely used in smaller railway stations in India. The request apparently arose out of the observation that a significant number of them, designed on the basis of existing foreign codes, were failing although the specified dynamic pressure was quite high. By hindsight, one now knows that this observation was justified since recent studies (Davenport et al 1977) have clearly shown that wind loads at the corners and awnings on low-rise buildings are much higher than was originally estimated. The IISc measurements of pressure distribution on a model were made in smooth flow without simulating atmospheric turbulence, which is known to have a very significant effect on the pressure distribution on such structures. Nevertheless, the data proved to be helpful as testified by the very few, if any, reports of failures of redesigned shelters in recent times. There was a decade-long period of inactivity after this study before the investigation of the 


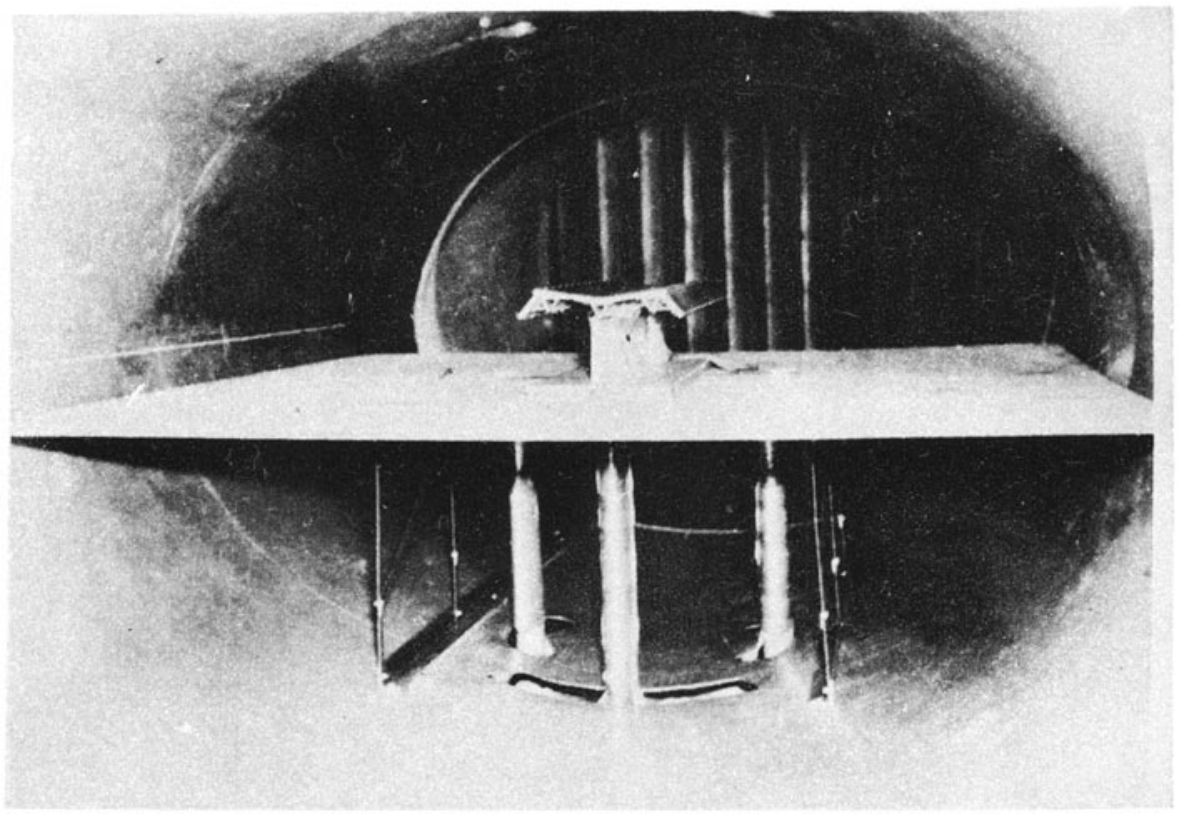

Figure 1. Model of railway platform shelter in the $2.25 \mathrm{~m} \times 1.5 \mathrm{~m}$ elhptic wind tunnel.

aerodynamic stability of the proposed $150 \mathrm{~m}$ high RCC chimney for the Badarpur Thermal Power Station (Rao et al 1970) was taken up, also at IISc. Since that time more than 50 projects have been handled at the IISc (table 1).

The availability of wind tunnels of reasonable size after 1970 stimulated studies in wind engineering at the Indian Institutes of Technology (IIT), at Kanpur, Delhi, Bombay and Madras, as well as at Roorkee and Anna Universities (table 2). However, more than $80 \%$ of wind engineering studies in India have been carried out at the IISc. The experimental programmes have covered almost all aspects of wind engineering, comprising tests on models of chimneys, cooling towers, TV towers, land transportation vehicles like automobiles and ships, dispersion of

Table 1. Types of model studies conducted in India.

Chimneys: Single and multiflue chimneys as well as several identical chimneys in a line

Cooling towers: Single and upto six in various types of clusters

Cable-stayed suspension bridges: Sectional models of both erection and completed sections, tower legs

TV towers: Force and aero-elastic models

Antenna dish: Pressure distribution and aero-elastic models.

Land transportation vchicles: Railway carriages, railway engines, automobiles, trucks and ships

Buildings: Low and high-rise buildings including effect of aerodynamic interference

Miscellaneous: Windmills, helmets, mountains etc 
Table 2. Major low speed wind tunnel facilities in India.

\begin{tabular}{ll}
\hline Facility & Location \\
\hline $4.25 \mathrm{~m} \times 2.75 \mathrm{~m}$ Open circuit & IS , Bangalore \\
$2.5 \mathrm{~m} \times 7.5 \mathrm{~m}$ Closed circuit & ISc, Bangalore \\
elliptic cross-cection & HAL' Bangalore \\
$2.75 \mathrm{~m} \times 1.8 \mathrm{~m}$ Closed circuit & NAL. Bangalore \\
$0.91 \mathrm{~m} \mathrm{dia} \mathrm{Open} \mathrm{circuit}$ & IIT. Kanpur \\
$0.9 \mathrm{~m} \times 0.6 \mathrm{~m}$ Closed circuit & IIT. Madras \\
$1.5 \mathrm{~m}$ dia Closed circuit & Roorkee University \\
$2.0 \mathrm{~m} \times 2.0 \mathrm{~m}$ Open circuit & DGCA. Technical Centre, \\
$1.25 \mathrm{~m} \times 1.5 \mathrm{~m}$ Open circuit & Delhi \\
\hline
\end{tabular}

* NAL - National Aeronautical Laboratory; ${ }^{\dagger}$ DGCA - Directorate General of Civil Aviation; + HAL - Hindustan Aeronautics Ltd.

pollutants etc. These studies and the experience of practising engineers indicated not only a need to revise some of the relevant Indian standards like IS-875 (1964) but also to study the nature of the Indian atmosphere in order to evolve more realistic wind speed specifications (Narasimha \& Shrinivasa 1984; Seetharamulu et al 1985, pp. 47-51; Rao 1985b, pp. 11-19; Sharma 1985). Coinciding with the increase in the general awareness of problems in wind engineering in India, a number of seminars and workshops have been held, whose proceedings have been published (Seminar 1980; Workshop 1984; Asia-Pacific Symposium 1985; Indo-US Workshop 1985) and the Department of Science and Technology (DST) formed a Committee to identify problem areas and suggest the support required to study them (DST 1983). It is appropriate at this stage to review the work that has gone on and identify possible areas for further study. We begin by a consideration of the Indian atmosphere insofar as wind loads on structures are concerned.

\section{Peculiarities of the Indian atmosphere in respect of wind loading analysis}

Historically, wind loading on a structure was viewed in the early days as simply one of estimating the 'dynamic pressure' of the wind at the site of the structure and multiplying it by the 'shape factor' (or drag coefficient) and the characteristic area of the structure to obtain the wind force. This was the view taken in the Indian Standard Specification IS-875 (1964). However, as the number of tall and slender structures increased, it became clear that not only the dynamic action of wind for 'along-wind' or drag-direction load has to be computed, taking into account the unsteady nature of the wind load, but also that other dynamic responses like vortex excitation, galloping, flutter and ovalling have to be examined. It then became necessary also to know the characteristics of the Indian atmosphere in greater detail than had been done earlier. The starting point for this study was to put the extreme wind and mean hourly wind data on a more scientific basis.

A number of extreme wind analyses have been made using both the FischerTippet type I (FT1) method or its variants (Rao 1985b, pp. 11-19), and the FischerTippet type II (FT2) method. It is now recognised that five mechanisms operate to create high winds in India (Rao 1985b, pp. 11-19), namely, tropical cyclones, extra-tropical cyclones, monsoons, local thunderstorms such as kal-baisakis in 
West Bengal and the pressure system. In most parts of India, there is a mix of the above population which is reflected in the curved line that one obtains when the reduced variate is plotted against the wind speed. It is well-known (Mayne 1979) that a linear regression will be obtained only if the mechanism is of a single type and will then correspond to the FTl type. For example, the extreme winds in Western Europe and Canada are caused by the fully developed pressure systems which result in a linear regression of reduced variate against wind speed. At present, available theories for calculating the loads on a structure (Davenport 1967; Vickery \& Basu 1984) implicitly assume the wind to be 'well-behaved', i.e., caused by a pressure system. In view of the fact that extreme winds at most of the locations in India are apparently caused by different mechanisms, it appears that a prediction based on FT2 is to be preferred (although it cannot be theoretically justified, but is simply a 'good fit' curve) than FT1. Nevertheless, a combination of FT1 analyses, local judgement and orography of India has yielded what is believed to be a more realistic extreme wind map for India (Sharma 1985).

Since a knowledge of existing probabilistic loading theories (Vickery \& Basu 1984) requires mean hourly wind (MHW) speed, an attempt was made by Seetharamulu et al (1985, pp. 47-51) to generate such data from information supplied by the India Meteorological Department (IMD). They observed the surprising result that the ratios of peak gust (PG) to MHW for practically all the stations in India were higher than the values quoted for regions with winds due to the pressure system (Davenport 1967). Their observation appears to have borne out a somewhat similar observation of Narasimha \& Shrinivasa (1984) that the ratio of peak gust to yearly average wind is higher in India than in the United States.

However, it is necessary to examine more closely not only the manner in which MHW is derived in India and many other countries but its definition as well. 'Mean hourly wind' of the India Meteorological Department is the mean wind during the last ten minutes of every hour and not the average during the entire hour. This is derived from an eye judgement of continuous wind data of the Dyne pressure tube anemograph in which the contribution of very short duration high winds cannot be given adequate importance in the integrated value. On the other hand, in those countries where the MHW is derived from some kind of integrating instruments such as a propeller or vane anemometer allowed to run continuously through the short duration peaks, one may expect a more realistic mean. Mani \& Mooley (1983) have reported examining the true full hour mean and the mean of the last ten minutes and find a $5 \%$ difference between them, but the basis of the two means is not indicated. More recently, Rao et al (1985b) have examined the effect of various averaging times in moderate winds at Balasore and concluded that a much larger time interval of $15-20 \mathrm{~min}$ is required to obtain nearly steady values. However, the observations of Narasimha \& Shrinivasa (1984) regarding the larger ratio of peak gust to yearly mean in India does suggest that the peak gusts are due to a different mix of populations than in the US since yearly means in IMD are obtained from the integrated values of the number of revolutions of the cup anemc therefore be taken as a true mean, in the sense that the contributi duration gusts are properly counted.

Recently, Rao (1987) has found that the peak MHW for Madras : : FT1 distribution but that the peak gust is significantly convex in the $\mathrm{FI}$; ,ure 2). Measurements of turbulence characteristics in the Indian at. ind may short ere was 


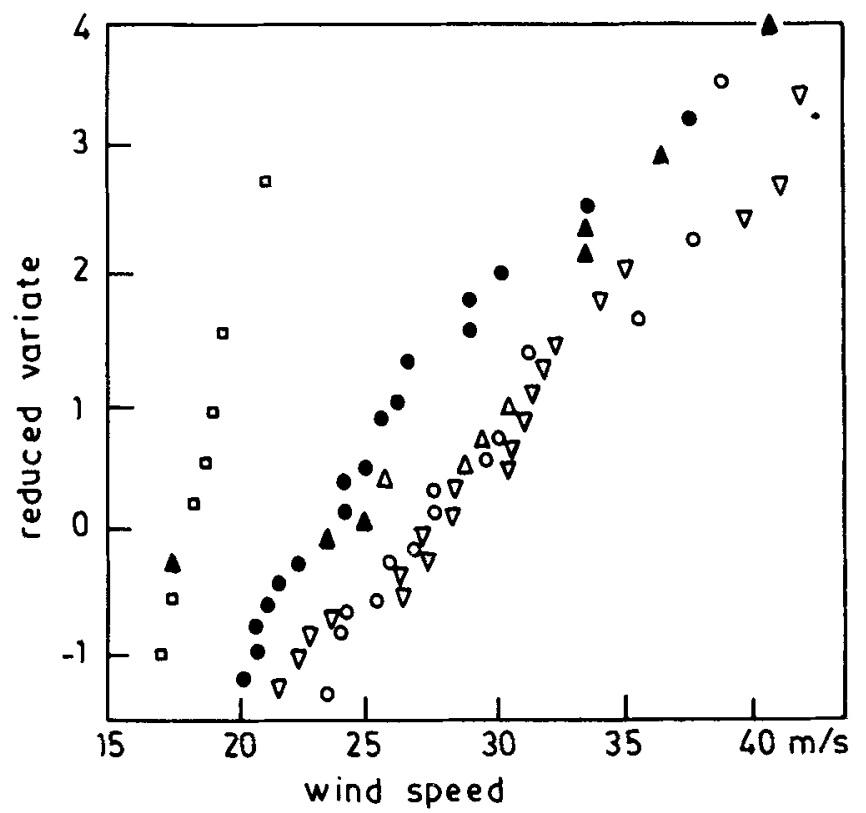

Figure 2. FTl plot of reduced variate vs, wind speed for PG and MHW. ( $\square-$ MHW; the rest of the symbols represent peak gusts.)

initially attempted using an instrumented Canberra aircraft (DRDO 1975). More recently boundary layer measurements in the first $30 \mathrm{~m}$ have been made (Rao et al 1985b). The earlier measurements suggested that the frequency of atmospheric gusts in India of a certain duration are more than that in European countries. The latter boundary layer measurements have been made in pressure system winds of fairly low magnitude and one hopes that data during the high winds and cyclones (or near cyclonic regions) will become available in course of time.

To sum up, one may state that the foundations for a rational study of wind characteristics in India of the nature that is useful for structural design are now being laid.

\section{Facilities}

Table 2 gives the major facilities in the form of wind tunnels that are available in India. Smaller wind tunnels are available in a few other places. The most extensively used facility has been the $4.25 \mathrm{~m} \times 2.75 \mathrm{~m}$ open circuit wind tunnel of the Department of Aerospace Engineering, IISc, Bangalore.

A major deficiency in most of the major facilities has been that they were not originally designed for wind engineering studies and as such it is quite difficult to reproduce the turbulence characteristics of the atmospheric boundary layer. The exception is the $2 \mathrm{~m} \times 2 \mathrm{~m}$ wind tunnel at Roorkee University in which, however, the maximum wind speed is limited at present to $20 \mathrm{~m} / \mathrm{s}$ with the tunnel empty.

\section{Theoretical studies}

Much of the attention in theoretical studies has been directed towards the interpretation of model studies to full scale. It appears that the effect of Reynolds 
and Strouhal numbers is generally to reduce the amplitude of response of most structures subjected to vortex-excited oscillations (Rao 1975). A linear analysis of the response of simplified models of structures, such as sectional models of suspension bridges or cantilever structures mounted as linear mode models, enables the prediction of the full scale response more accurately (Rao. 1972).

\section{Model studies}

\subsection{Modelling considerations}

By far the greatest effort has been in the area of model studies. Table 1 gives a list of these studies. It is well-known that a number of non-dimensional parameters have to be satisfied to ensure that model results are a replication of the response of the full-scale structure. These are:

(a) geometric similarity;

(b) Reynolds number $\left(U_{0} l / \nu\right)$;

(c) stiffness parameter $\left(E I / \rho U_{0}^{2} l^{4}\right)$;

(d) density ratio $\left(\rho_{s} / \rho_{a}\right)$;

(e) structural damping $(\delta)$;

(f) ratio of velocity of exit gases to free stream velocity;

(g) ratio of wall thickness to diameter;

(h) characteristics of the atmosphere: All the average and fluctuating components, and length scales, suitably scaled down. If the above non-dimensional parameters are satisfied, then the relation between the following model response parameters and that of the full-scale structure can' be shown to be as follows:

(i) equality of Strouhal number: $n l / U_{0}$;

(ii) equality of non-dimensional amplitude response, both static and dynamic; (iii) stress at any point, static and dynamic at the same free stream velocity.

It is well-known that it is practically impossible to satisfy all the requirements of dynamic similitude, in particular the Reynolds number ( $R e$ ), and it is usual to develop or follow certain practices to extrapolate model results to full scale taking into consideration the deviations from strict similarity considerations. The practice adopted at the IISc is described later insofar as Re effect corrections are concerned. Although mean velocity profiles have been simulated, using Cowdrey's (1967) method (figure 3 shows the grid rods), simulation of atmospheric turbulence has not yet been done due to the short upstream fetch in most of the existing wind tunnels. The issue of both the mean velocity profile and turbulence structure is complicated by the knowledge that available information refers to well-behaved winds or winds due to pressure system while extreme winds in practically all parts of India are, as explained earlier, due to some kind of cyclone or thunderstorm.

\subsection{Modelling practices}

Depending on the nature of the problem to be studied, models are categorised as (1) rigid models, (ii) aeroelastic models, (iii) linear mode models, (iv) sectional models, and (v) distorted models (Rao 1985a). The practices adopted in the case of models of some of the important structures are described below. 


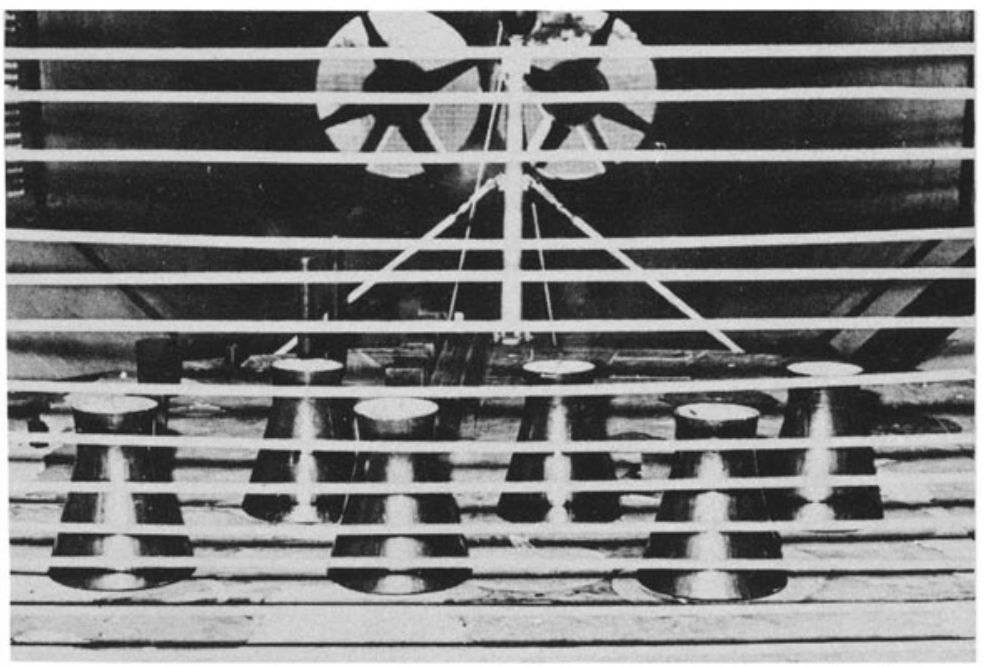

Figure 3. Parallel rods for generating mean velocity profite in a wind tunnel.

\subsection{Chimney models}

The usual practice in making chimney models is to make them as slightly distorted aeroelastic models. The models are made in aluminium by accurately boring on the inside and machining on the outside but the thickness is also geometrically scaled. This means that the models are not exactly replicated full scale chimneys but it is not difficult to work out the relation between a truly replicated model and an aluminium model, in the case of RCC and steel chimneys. Table 3 gives the relation between such models and full scale structures. The model is deliberately made smooth for reasons which will be explained later. Figure 4 shows a number of these aluminium models.

Table 3. Relation between geometrically similar and fully acroelastic models.*

\begin{tabular}{|c|c|}
\hline Property & Full-scale value \\
\hline Frequency & $\left(n_{m} / N\right)\left(E_{/} / E_{m}\right)\left(\rho_{m} / \rho_{f}\right)^{1 / 2}$ \\
\hline $\begin{array}{l}\text { Critical wind specd for vortex } \\
\text { excited oscillation (velocity } U_{f} \text { ) }\end{array}$ & $U_{m}\left(E_{f} / E_{m}\right)\left(\rho_{m} / \rho_{f}\right)^{1 / 2}$ \\
\hline Oscillatory amplitude & $N\left(\rho_{m} / \rho_{f}\right)^{1 / 2}$ \\
\hline $\begin{array}{l}\text { Streamwise deflection, } W_{f} \text {, at the } \\
\text { same dynamic pressure }\end{array}$ & $N\left(E_{m} / E_{f}\right) W_{m}$ \\
\hline $\begin{array}{l}\text { Oscillatory moment at resonance } \\
\text { at any point, } M_{\omega}\end{array}$ & $N^{3}\left(\rho_{t r} / \rho_{f}\right)$ \\
\hline $\begin{array}{l}\text { Along-wind moment at any point at } \\
\text { the same dynamic pressure }\end{array}$ & $M_{d m} N^{3}(0 \cdot 8 / 1 \cdot 2)$ \\
\hline
\end{tabular}

* Subscript ' $f$ ' denotes full scale value and subscript ' $m$ ' the model value. $\boldsymbol{N}=$ model scale, more than $1 \cdot 0$.

${ }^{\dagger}$ Full scale $C_{D}$ is takeı as 0.8 and that of model 1.2 . 


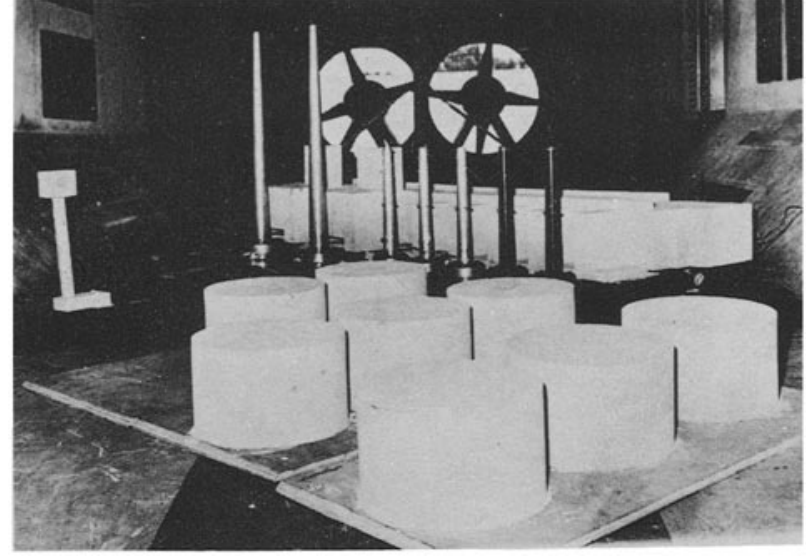

Figure 4. Cluster of aluminium chimney models.

\subsection{Natural draught cooling tower models}

Aluminium has also been used to make aeroelastic models of natural draught cooling towers. A particularly strong wood known as Irumbuli which is said to have a specific gravity of almost $1 \cdot 5$, is used as the former to spin thin aluminium sheets. By taking a sheet which is slightly thicker than the scaled-down value (or choosing the scale to suit a slightly thicker commercially available aluminium sheet), it was possible to obtain models which were accurate to within $5 \%$ in thickness and better than $1 \%$ in other dimensions. Figure 5 shows a group of such models.

\subsection{Models of framed structures}

Models of framed steel structures were made by choosing or getting rolled sheets of geometrically scaled-down thicknesses and bending them to the shape of the angle etc. Soldered joints were found to be unsatisfactory and hence all joints were silver-brazed. The same procedure is used in the case of aeroelastic models of antennae.

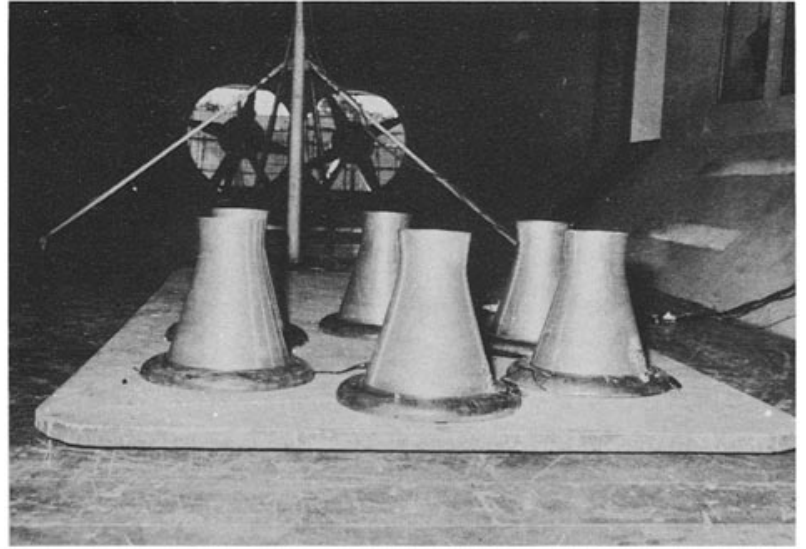

Figure 5. Aluminium aeroelastic models of natural draft cooling towers. 


\subsection{Sectional models of suspension bridges}

Sectional models of suspension bridges are made by using both wood and steel sheets. A similar procedure is adopted in the case of linear mode models, such as those of the service towers for satellite launch vehicles (figure 6).

\subsection{Damping of models}

It is necessary during a test programme to vary the structural damping of models to overlap the expected value of the full-scale structure: This is done in the case of earth-fixed models like chimneys and linear mode models of satellite launch vehicles by mounting them on neoprene rubber pads of different thicknesses. In all other cases, oil dashpots are used.

\section{Examples of model studies}

\subsection{Chimneys and towers}

A number of studies (exceeding 30 ) of chimneys and towers have been carried out and these studies have yielded many useful results. It has been found that in the case of chimneys, it is more economical to use aerodynamic remedial measures when the dynamic moments due to a vortex or other modes of excitation at their natural frequency, exceed the direct wind (or 'along-wind') moment by more than about 10\% (Krishnaswamy et al 1975, pp. 209-215; Rao et al 1985a; Rao 1985c, pp. liii-Ixiv). A very effective remedial measure has been the development of discrete strakes (figures $7 \& 8$ ) which has been incorporated in more than 20 chimneys in India and abroad. It is observed that the additive drag penalty of discrete strakes is much less than that of continuous strakes (figure 9).

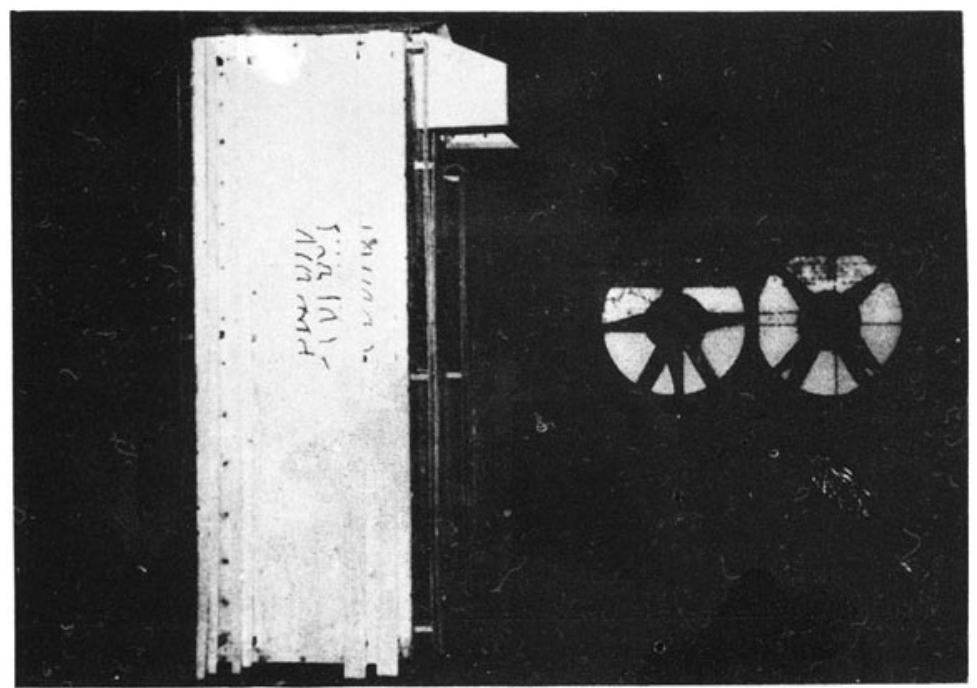

Figure 6. Linear mode model of service tower of satellite launch vehicle. 

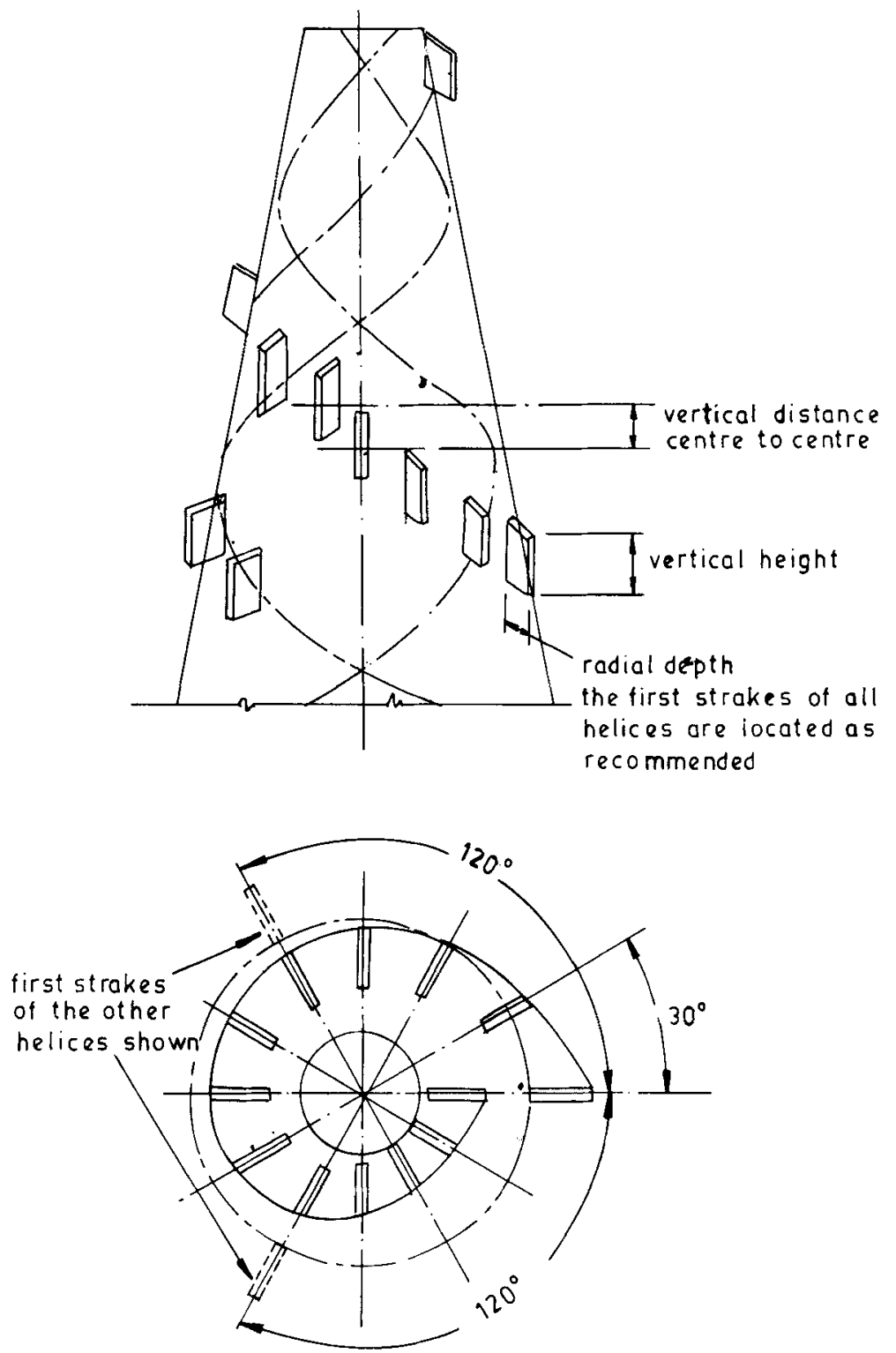

Figure 7. Geometric configuration of discrete strakes.

The study of the effect of aerodynamic interference between chimneys continues to attract worldwide attention (Price \& Paidoussis 1984; Vickery 1981; Krishnaswamy et al 1975, pp. 209-215; Lakshmana Gowda \& Prabhu 1987) but it must be stated that the exact nature of the enhanced oscillatory loading and the effect of Reynolds number are not yet fully understood. There is however no dispute over the fact that aerodynamic interference between chimneys does lead to increased oscillatory response. Figure 10 shows the result of one such study at IISc which suggests that the enhancement can be as much as 10 times in subcritical $\operatorname{Re}$ flow. The remedial measures described earlier in the form of discrete strakes work equally well in substantially alleviating increased oscillatory amplitude. The effect 


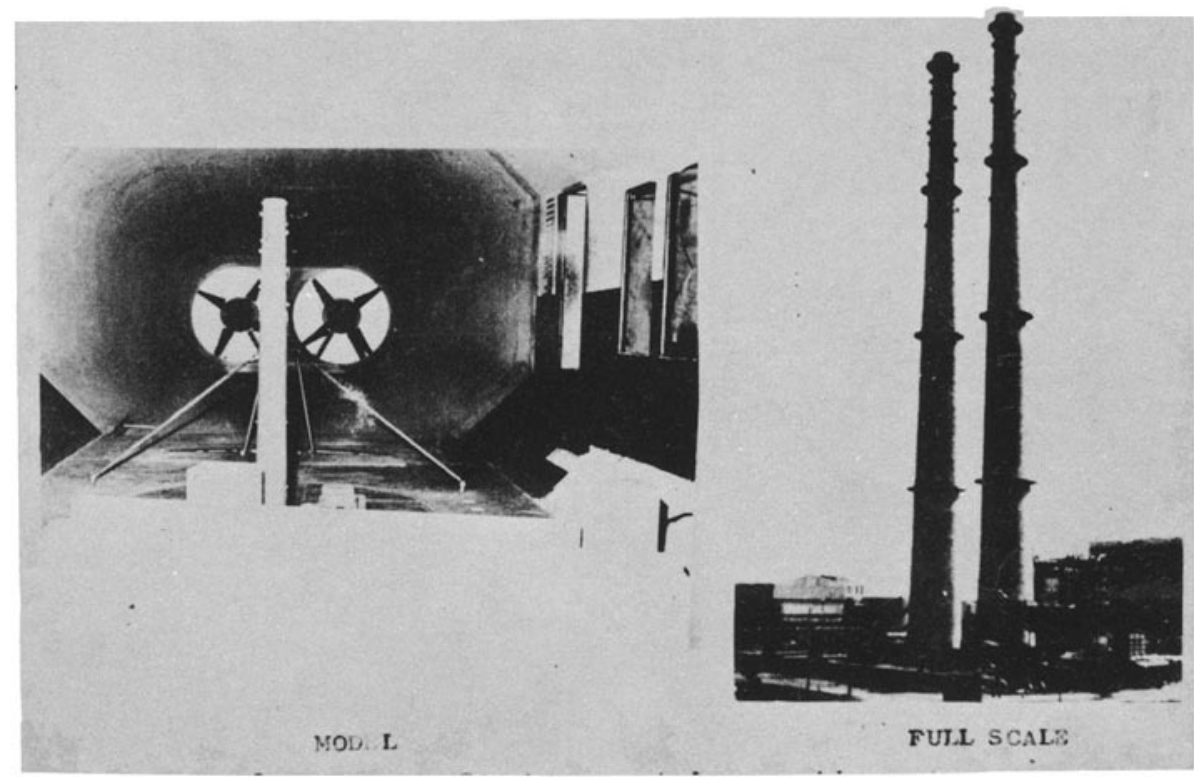

Figure 8. Discrete strakes on wind tunnel models and full scale chimneys

of wind direction on a cluster of five chimneys is shown in figure 11 . It is seen that a wind which is slightly inclined to the line of chimneys (by about $15^{\circ}$ ) gives rise to maximum load enhancement due to oscillation and the most severely affected one is the second one in the windward direction. In a study of the effect of the surrounding structures in a thermal power station, Swamy \& Lakshmana Gowda (1983) found that they tended to reduce the wind load on chimneys. Prem Krishna et al (1983) found that aerodynamic damping of the chimney increases with increased wind speed even in subcritical flow.

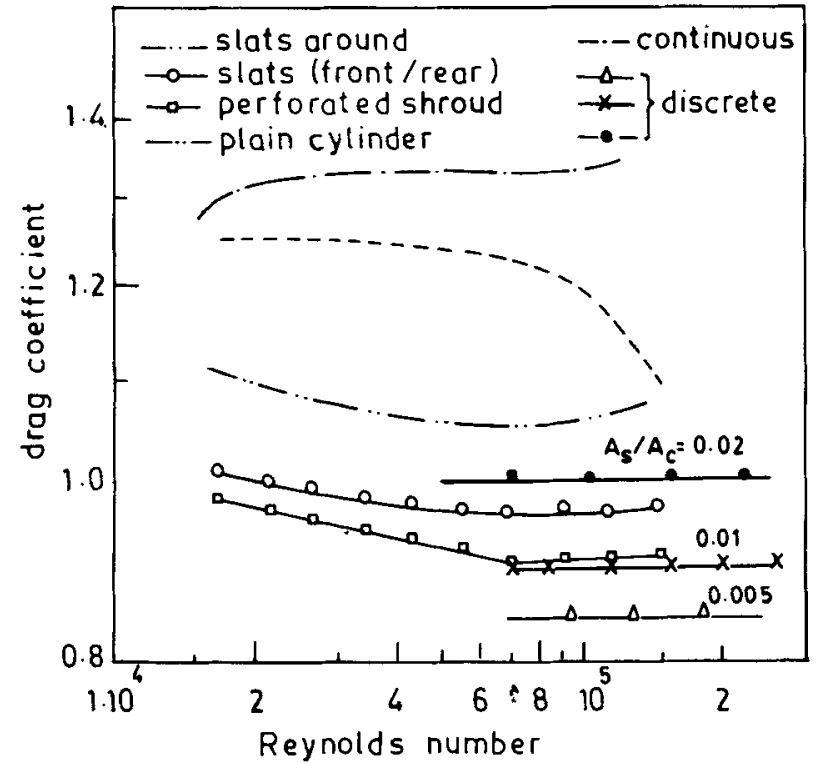

Figure 9. Total drag of chimney cross-section with acrodynamic remedial measures including continuous and discrete strakes. 


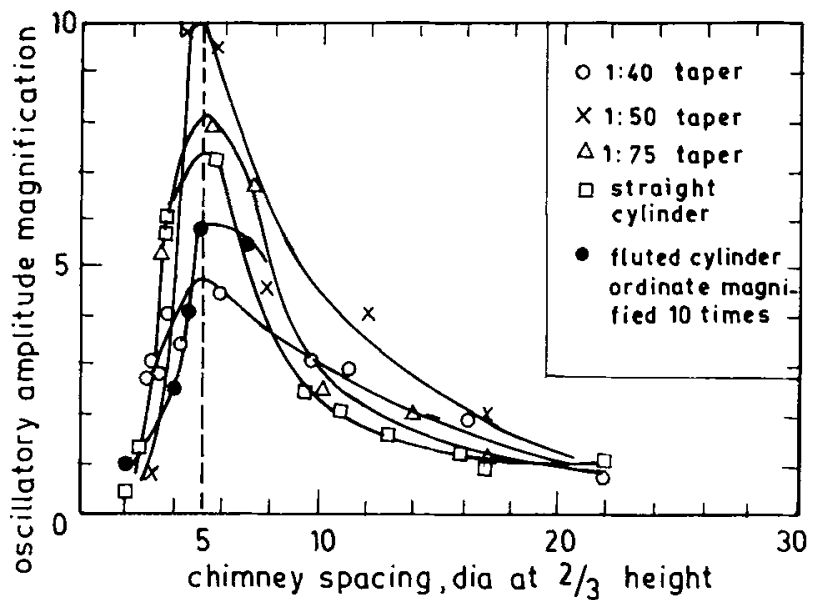

Figure 10. Effect of acrodynamic interlerence on oscillatory amplitude of lecward chimney.

Experimental and theoretical estimates of the gust factor for an unsymmetrical tower were carried out by Nigam et al (1981) who found that the gust factors in two perpendicular directions were substantially different, being $2 \cdot 2$ and $3 \cdot 2$.

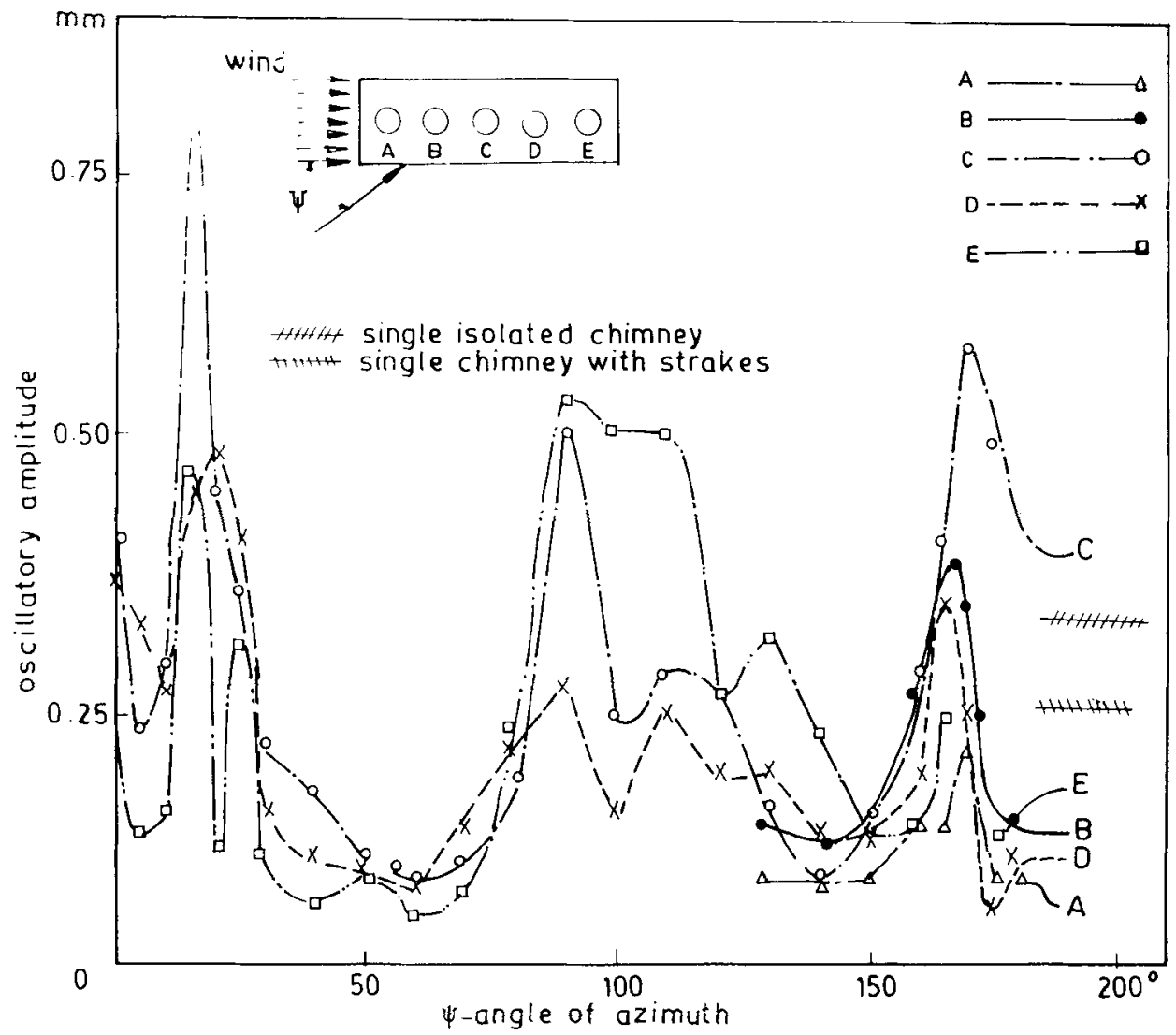

Figure 11. Effect of wind direction on amplitude enhancement. 


\subsection{Cooling towers}

Tests on various planform configurations of cooling towers have been carried out on pressure distribution models to assess the effect of planform geometry (Rao \& Reddy 1980). The load enhancement was found to vary from 1.4 to $2 \cdot 0$. Aeroelastic models of cooling towers have also been tested to determine tendencies for ovalling oscillations. It is found that critical loading due to aerodynamic interference takes place when the wind is inclined at an angle of about $45^{\circ}$ to the line of cooling towers.

\subsection{Cable-stayed suspension bridges}

Sectional models of cable-stayed suspension bridges have been tested (Krishnaswamy et al 1971, pp. 678-698; Krishnaswamy 1981). Tests on linear mode models of the towers in their free standing mode have been made to assess galloping tendencies. When the cross-sectional shape was rectangular, with the bottom open, a set of discrete strakes was found to be very effective in suppressing model oscillations (figure 12) and increasing the critical wind speed for the onset of flutter (Krishnaswamy et al 1971, pp. 678-698). But this device did not work when the bridge had a nosing. So far, no aerodynamic remedial measure in the form of some flow-spoiling device has been found which will suppress galloping instability of square cross-sections.

\subsection{Low-rise structures}

A few studies of wind effects on low-rise structures have been made in simulated mean winds (Krishnaswamy et al 1971, pp. 678-698, 1982; Gupchup \& Agashe 1981). The aim was to obtain mean pressure data.

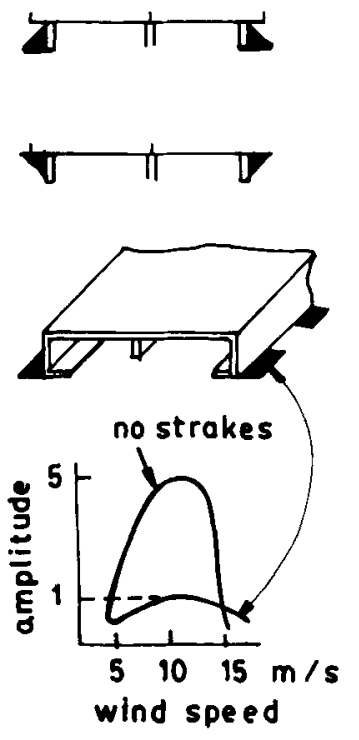

Figure 12. Effect of discrete strakes on oscillations of cablestayed bridges. 


\subsection{Extrapolation of model results to full scale conditions}

Considerable thought has been applied to clarify the extrapolation of model results to full-scale conditions both for along-wind load and across-wind load. The extrapolation obviously depends on the type of body and the approach adopted in the case of some typical bodies is given below. The dominant scaling parameter is the Reynolds number.

5.5a Sharp edged bodies: If the test Reynolds number exceeds 25,000 , the model values are taken to be valid at full scale $\mathrm{Re}$. This requirement is achieved in all test programmes so far.

5.5b Along-wind force-rounded bodies: Along-wind force on rounded bodies such as circular cylinders are estimated by conducting model studies with either known laminar boundary layer separation or by fixing transition with a trip wire (Swamy \& Lakshmana Gowda 1983). Every effort is made in both cases to ensure that conditions corresponding to a region in the 'drag bucket' do not exist and the model surface is therefore made smooth. A 'proportionality factor' is then used to obtain full-scale value as follows:

$$
\frac{\text { full-scale force coefficient }}{\text { model force coefficient }}=\frac{\text { full-scale circular cylinder force coefficient }}{\begin{array}{l}
\text { full-scale circular cylinder force } \\
\text { coefficient at transcritical } R e
\end{array}} .
$$

The RHS terms are obtained from handbooks such as ESDU (1978). This procedure is believed to be more logical than the ESDU procedure of "difference techniques', namely full-scale force coefficient $=$ model force coefficient - (fullscale circular cylinder force coefficient at full-scale $\mathrm{Re}$-circular cylinder force coefficient at model $\mathrm{Re}$ ).

5.5c Dynamic response - rounded bodies: The estimation of full-scale dynamic conditions from model results is more complicated. Corrections have to be applied for differences both in Strouhal number ( $\mathrm{St}$ ) and Reynolds number $(\mathrm{Re})$. Here, the known trends in Re vs. St and Re vs. oscillatory lift coefficient for circular cylinders are used (figures $13 \& 14$ ). As may be observed, there is no significant variation in oscillatory lift coefficient with $\mathrm{Re}$, if care is taken to ensure that there is no "lift bucket'. Hence, if tests are carried out on a smooth rounded body at an Re lying to the left of the oscillatory lift and drag buckets, one may expect the full-scale

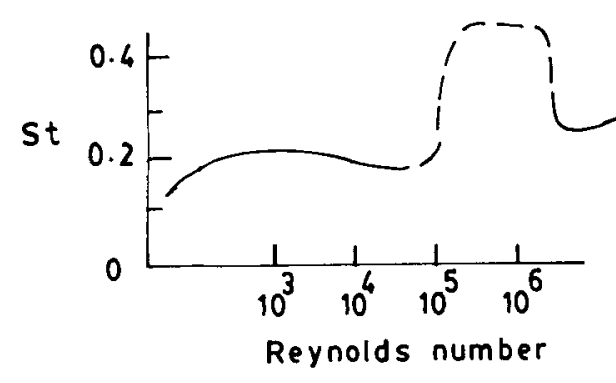

Figure 13. Strouhal number-Reynolds number relation for circular cylinder. 


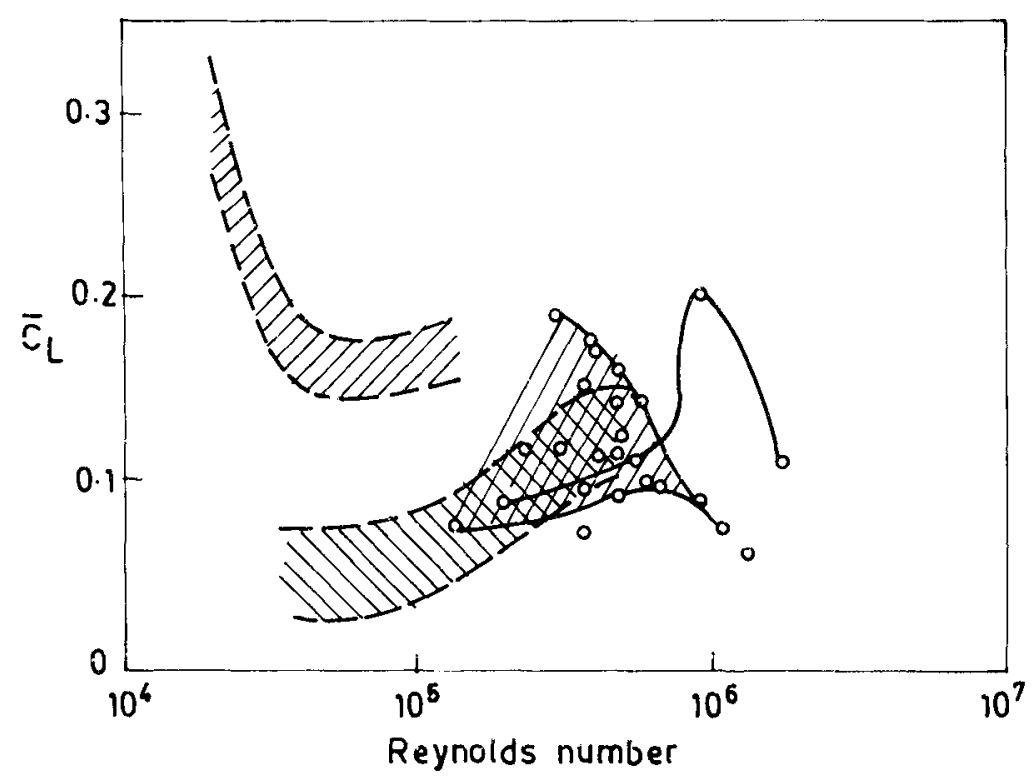

Figure 14. Oscillatory lift coefficient of circular cylinder.

oscillatory lift coefficient to be perhaps the same as that of the model value. Together with the known gradual and practically linear rise of St vs. Re from subcritical to transcritical $\mathrm{Re}$ (again excluding the 'inverted bucket region' of $\mathrm{Re}$ from $2 \times 10^{5}$ to $2 \times 10^{6}$ ), one arrives at the following simple rules of extrapolation of model results to full scale.

$\frac{\text { Full scale } \mathrm{St}}{\text { Model St }}=\frac{\mathrm{St} \text { of circular cylinder at full scale } \mathrm{Re}}{\mathrm{St} \text { of circular cylinder at model } \mathrm{Re}}$

and

non-dimensional model transverse oscillatory response $=$ nondimensional fuil-scale transverse oscillatory response.

\section{Full-scale measurements}

It is unfortunate that no systematic full-scale measurements were made on any structure in India till about 1986. The unusual lighting towers of the Jawaharlal Nehru Stadium at New Delhi have now been instrumented (figure 15) and plans are under way to instrument a few chimneys and cooling towers. One hopes that these measurements will not only throw light on those properties of the wind relevant to wind engineering but also lead to better methods of wind-loading analysis.

\section{Conclusions}

As stated earlier, it is believed that foundations for serious studies on wind engineering have been laid in India. A great deal more still needs to be done of 

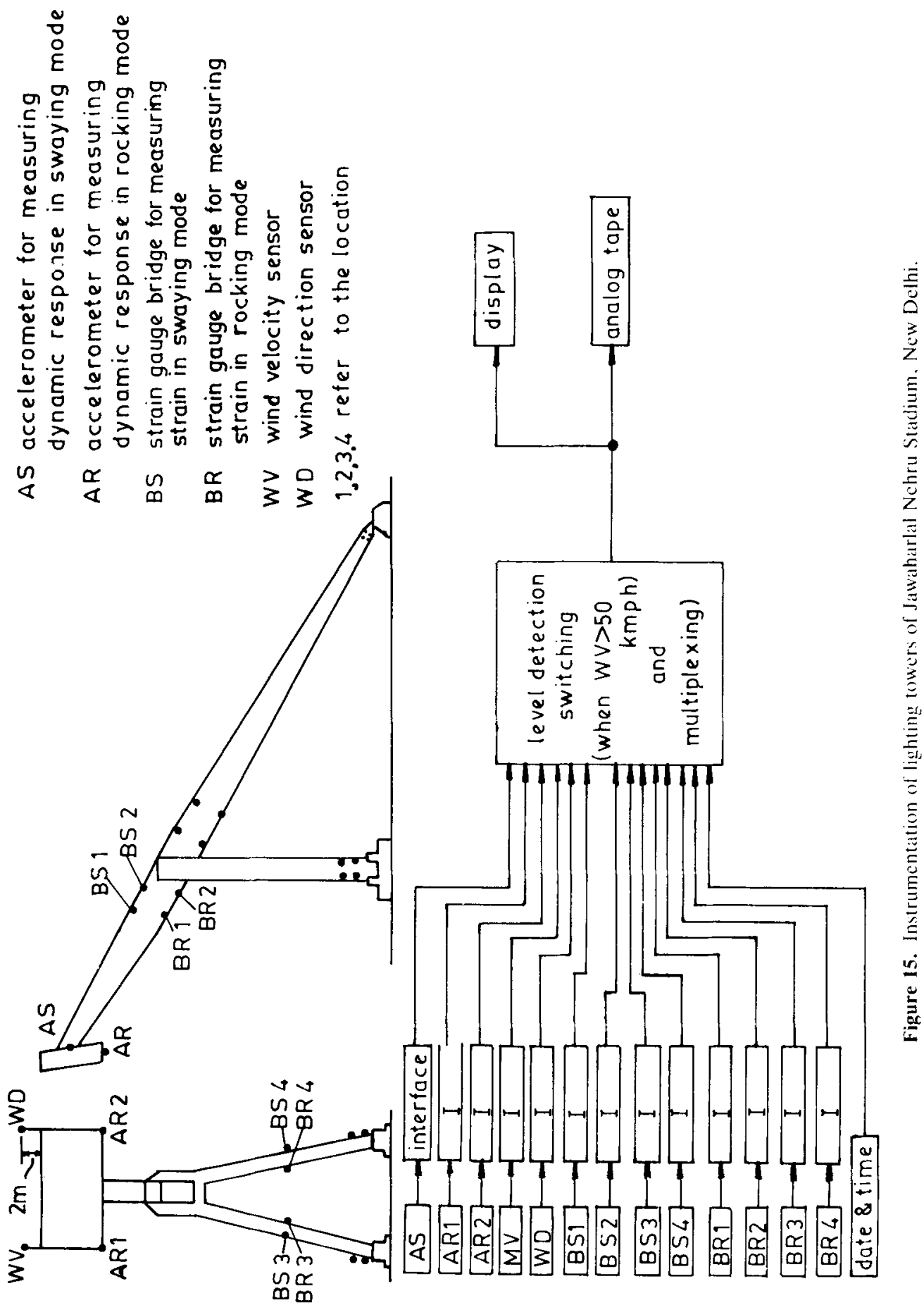
which the following appear to be most important in the foreseeable future. (i) Increase the number of Dyne pressure tube anemograph stations to at least 150 , develop and install microprocessor-based wind speed and direction sensors with sufficient memory for storing the more important peak and average data.

(ii) A larger number of wind engineering wind tunnels are required; this includes facilities for creating cyclonic conditions.

(iii) More full-scale measurements on both tall- and low-rise structures have to be made. In particular, greater emphasis on the study of aerodynamic interference should be made.

(iv) It is necessary to strengthen theoretical studies of both the meteorological data on wind loads and the dynamic action of the wind.

The foundations that have been laid have already demonstrated visibly the returns in terms of more economical designs and creation of more reliable databases. The future must build on these.

\section{References}

Asia-Pacific Symposium 1985 Asia-Pacific Simposium on wind engincering (Meerut: Sarita Prakasan) Cowdrey ( F 1967 A simple method for the design of wind tunnel velocity profile grids. NPL. Aero note 1055. England

Davenport A ( 1967 J. Siruct. Dive, Am. Soc. (iv. Eng. 93: ST3

Davenport A (3. Surrey D. Stathopoulos $T 1977$ Wind loaks and kow-rise buildings:- Final repon of phases $I$ and II. BLWT-SSS. University of Toronto, Western Ontario

DRDO 1975 Report on the measurement of atmospheric turbulence in India using an instrumented Canberra aircratt. Report of the Defence Research and Developrnent Organisation, New Delhi

DST 198.3 Report of the (ommitce on wind engincering. Department of Science and Technology. New Delhi

ESDU 1978 Fluid mechanics (London: 1SDL :) vol. 1-3

Gupchup V N, Agashe S N 1981 J. Inst. Eng. (India) (Spec. 1ssuc) 61: 94-97

IS-875 1964 Indian Standards Specification (New Delhi: Indian Stand. Inst.)

Indi-US Workshop 1985 Indo-LS workshop on wind disaster mitigation. Procededings. Structural Enginecring Research Centre, Taramani, Madras

Krishnaswamy T N 1981 J. Inst. Eng. (India) (Spec. lssue) ND2 61: 43-50)

Krishnaswamy T N. Rao A K. Rao G N V. Durvasula S, Reddy K R 1971 Proc. III 1nt. Conf. on wind effects on buildings and structures (Tokyo: University Press)

Krishnaswamy T N. Rao G N V. Durvasula S. Reddy K R 1975 Proc. IV Int. Conf on wind effects on buildings and structures, Heathrow (London: Pergamon Press)

Krishnaswamy $T$ N. Rao G N V. Durvasula S, Reddy K R 1982 Wind tunnel model study of $14 \mathrm{~m}$ dia parabolic antenna dish of the Space Application Centre, IWTR 164, Department of Aerospace Enginecring. Indian Inst. Sci., Bangalore

Lakshmana Gowda B H. Prabhu D R 1987 J. Solund Vibr. 112: 487-502

Mani A. Mooley D A 1983 Wind energy data for India (New Delhi: Allied Publishers)

Mayne J R 1979 J. Ind. Aerodyn. 5: $109-1,37$

Narasimha R, Shrinivasa U 1984t Sadhana 7: 259-274

Nigam N C, Gupta A K, Mathur S P 1981 J. Inst. Eng. (India) (Spec. Issue) ND2 61: 69-76

Prem Krishna, Badruddin Ahmed. Pande P K 1983 Proc. 6th. Int. Conf. on wind engineering vol. 3

Price S J, Paidoussis M P 1984 J. Wind Eng. Ind. Aerodyn. 3: 329-348

Rao G N V 1972 J. Aeronaul. Soc. India 24: 242-245

Rao G N V $1975 / /$ US Natl. Conf. on wind engineering res'arch (University of Colorado Press) paper JV-8-1

Rao G N V 1985a J. Inst. Eng. (India) AE 1\&2 65: 11-I8

Rao G N V 1985h Proc. Asia-Pacific Symp. on wind engineering (Meerut: Sarita Prakashan)

Rao G N V 1985c Proc. Asia-Pacific Symp. on wind engineering (Mecrut: Sarita Prakashan) 
Rao G N V 1987 Proc: US-Asia Conl. on EMNHD, Banghok. Thailand (Bangkok: Asian Inst. Technol.) pp. A12-1-A12-s

Rao G N V. Durvasula S, Reddy K R 1985a Natl. Sem. on Tall RCC chimneys. (New Delhi: Natl. Thermal Power Corp.)

Rao G N V. Durvasula S, Reddy K R, Palaniswamy S 1970 Final report on model tests of $15 \mathrm{~m}$ high smoke stack for Badarpur Thermal Power Station, IWTR 84, Dept. of Aerospace Engineering, Indian Inst. Sci., Bangakre

Rao G N V. Redòy K R 1980 Proc. I Asian Cong. Fluid Mechanics (Bangalore: Asian Cong. Fluid Mechanics) vol. C. Paper B36

Rao K N. Prabhu A. Narasimha R 1985b Averaging time for atmospheric turbulence data, Report 85 ASE, Centre for Atmospheric Studies, Indian Inst. Sci.. Bangalore

Seetharamulu K, Swamy B L P, Chaudhry K K 1985 Proc. Asia-Pacific Symp. on wind engineering (Meerut: Sarita Prakashan)

Seminar 1980 Seminar on Industrial Aeronautics, Proceedings, Published by Institute of Engineers, India. ND2; vol. 61

Sharma M C 1985 Indo-US Workshop on Wind disaster mitigation, Proceedings, Structural Engincering Research Centre, Taramani. Madras

Sharma N C 1985 Indo-US Workshop on Wind disaster mitigation, Proceedings, Structural Engineering Research Centre, Taramani, Madras

Swamy N V C, Lakshmana Gowda B H 1983 Wind Tunnel Studies on the Model of the RCC chimneys for Korba West Bank Thermal Power Station. Madhya Pradesh, Report 117, Department of Applied Mechanics, Indian Inst. Technol., Madras

Vickery B J 1981 J. Wind Eng. Ind. Aerodyn. 1: 177-193

Vickery B J, Basu R I 1984 Eng. Struct. 6: 324-330

Workshop 1954 Workshop on wind effects on structures, organised by Inst. of Enginecrs (India). Selected papers published in vol. 65-66 of Acro Eng. Div.. J. Inst. Eng. (India) 